\title{
A Clinical Review of Ventricular Arrhythmias in Patients with Congestive Heart Failure
}

\author{
Authors: \\ Ali B.A.K. Al-Hadithi, ${ }^{*}$ Noel G. Boyle ${ }^{2}$ \\ 1. School of Clinical Medicine, Addenbrooke's Hospital, University of Cambridge, \\ Cambridge, UK \\ 2. UCLA Cardiac Arrhythmia Center, UCLA Health System, David Geffen School of \\ Medicine at UCLA, Los Angeles, California, USA \\ *Correspondence to NBoyle@mednet.ucla.edu
}

Disclosure: $\quad$ The authors have declared no conflicts of interest.

Received: $\quad 27.08 .18$

Accepted: $\quad 10.01 .19$

Keywords: $\quad$ Heart failure, ventricular arrhythmias, ventricular tachycardia (VT).

Citation: $\quad$ EMJ Cardiol. 2019 [Epub ahead of print]. DOI/10.33590/emjcardiol/18-00058R1

\begin{abstract}
Heart failure is an increasingly prevalent condition, which is associated with ventricular arrhythmias. The reduction in cardiac pumping efficiency leads to the activation of several compensatory mechanisms. These mechanisms eventually lead to cardiac remodelling and a decline in haemodynamic status, contributing to the formation of a substrate conducive to arrhythmias, including increased automaticity, triggered activity, and, most commonly, re-entry circuits. In turn, ventricular arrhythmias can lead to the worsening of heart failure. A diagnosis of heart failure and ventricular arrhythmias is obtained using the patient's history, examination findings, and investigation results. A key tool in this is echocardiogram imaging, which visualises the cardiac chambers, determines ventricular ejection fraction, and identifies structural abnormalities. A reduction in ejection fraction is a significant risk factor for the development of ventricular arrhythmias. Arrhythmias are diagnosed by ECG, Holter monitoring, and telemetry or event monitoring, and should initially be treated by optimising the medical management of heart failure. Anti-arrhythmic drugs, including beta-blockers, are usually the first-line therapy. Sudden cardiac death is a significant cause of mortality in heart failure patients, and implantable cardioverter defibrillator devices are used in both primary and secondary prevention. Antiarrhythmic drugs and catheter ablation are important adjunctives for minimising shock therapy. In addition, autonomic modulation may offer a novel method of controlling ventricular arrhythmias. The objective of this review is to provide a practical overview of this rapidly developing field in relation to current evidence regarding the underlying pathophysiology, burden of disease, and management strategies available.
\end{abstract}

\section{INTRODUCTION}

Pump failure and sudden cardiac death are the leading causes of death in heart failure patients; sudden cardiac death accounts for $30-50 \%$ of deaths. ${ }^{1,2}$ Heart failure increases the risk of sudden death by 6-9 times and most cases are the result of ventricular arrhythmias, ${ }^{3}$ which include ventricular tachycardia (VT) and ventricular fibrillation (VF).

Ventricular arrhythmias also cause significant morbidity and mortality, and heart failure patients have an increased burden of ventricular arrhythmias, particularly in advanced disease. ${ }^{2}$ 
Indeed, non-sustained VT is found in $20-80 \%$ of patients with heart failure. ${ }^{2}$ Ventricular arrhythmias manifest as palpitations, dyspnoea, dizziness, syncope, and sudden cardiac death. In addition, ventricular arrhythmias may lead to the progression of heart failure.

Implantable cardioverter defibrillator (ICD) devices are the principal intervention for primary and secondary prevention of sudden cardiac death in heart failure patients; 4,5 however, there is increasing evidence supporting the use of pharmacological agents and catheter ablation to minimise ICD shocks.

\section{EPIDEMIOLOGY}

An estimated 26 million people worldwide have been diagnosed with heart failure and it accounts for $>1$ million hospitalisations annually in Europe and North America. ${ }^{6}$ The overall prevalence of heart failure is $1-2 \%$ in the Western world, with most patients $>50$ years old. The prevalence increases sharply with age, affecting $>10 \%$ of those aged $\geq 85$ years. ${ }^{7}$ Annual mortality varies according to the severity of heart failure, although the relative proportion of sudden deaths is substantial across all classes. The yearly mortality rate from sudden death is 12-15\% within the New York Heart Association (NYHA) functional class I and II, while class IV has a sudden death rate of $50-60 \%$. Sudden death accounts for $50-60 \%$ of all deaths in NYHA functional class I and II, while class IV has a rate of $20-30 \%$.

Ventricular arrhythmias have been presumed to be the cause of a significant proportion of sudden deaths; other causes include bradyarrhythmias and pulseless electrical activity. ${ }^{9,10}$ Given their spontaneous nature, the prevalence of ventricular arrhythmias in heart failure patients is difficult to assess; however, ventricular arrhythmias have been noted to be particularly prevalent in patients with reduced ventricular ejection fraction and underlying cardiac ischaemia." The risk of ventricular arrhythmias is further increased by coexisting comorbidities, including obstructive sleep apnoea, hypoxaemia, electrolyte disturbances, catecholamine excess, pro-arrhythmic drug effects, hepatic dysfunction, and renal dysfunction. ${ }^{12}$ While ventricular arrhythmia encompasses several rhythm disturbances, it is important to note that VF and sustained VT have a higher disease burden and risk of sudden death compared to non-sustained VT and premature ventricular contraction.

\section{PATHOPHYSIOLOGY}

Heart failure occurs due to a reduction in the pumping efficiency of the organ as a result of myocardial injury. The most common cause of myocardial injury is ischaemic heart disease, although structural damage may also occur as a result of hypertension, diabetes, valvular disease, cardiomyopathies, infections, and toxin exposure (e.g., alcohol and chemotherapeutic agents).

Several compensatory mechanisms are activated in response to cardiac injury, including the Frank-Starling mechanism, neurohormonal activation, and ventricular remodelling. Although initially beneficial, these compensatory mechanisms eventually lead to an exacerbation of haemodynamic instability and thereby encourages further compensatory mechanisms, which produces a cycle of worsening heart failure..$^{13}$ Changes in the electrical and mechanical function of the heart, including fibrosis and regional hypertrophy, can predispose a patient towards the development of ventricular arrhythmias. ${ }^{14}$ The primary mechanisms for initiating and perpetuating ventricular arrhythmias include abnormal automaticity, increased triggered activity, and development of re-entry circuits, which is the most common mechanism. ${ }^{15}$ The electrical instability in ventricular arrhythmias can lead to haemodynamic collapse and consequent sudden death. Ventricular arrhythmias can degenerate to asystole.

It is important to note that there is a complex interplay between heart failure and ventricular arrhythmias (Figure 1). ${ }^{16}$ While heart failure can lead to the development of a substrate conducive to arrhythmia formation, ventricular arrhythmias may in turn accelerate the pathological mechanisms of heart failure. ${ }^{16} \mathrm{~A}$ 2006 study $^{17}$ found that appropriate shocks for ventricular arrhythmias in advanced heart failure were associated with a significant increase in pump failure death. It was unclear whether the shocks were the cause of the adverse outcomes or if they were a marker of disease progression; however, the 2013 ALTITUTIDE survival by rhythm study found that the mortality risk was associated with the underlying rhythm (either 
ventricular tachycardia or atrial fibrillation) rather than the shock therapy itself. ${ }^{18}$ There was no significant difference in survival between no shocks and inappropriate shocks for sinus tachycardia or noise/artefact/oversensing. In comparison, patients who received their first shock for monomorphic VT (hazard ratio: 1.65; $\mathrm{p}<0.0001$ ) or VF/polymorphic VT (hazard ratio: $2.10 ; \mathrm{p}<0.0001$ ) had a significantly higher mortality compared to patients who did not receive shocks. ${ }^{18}$ These results suggest that the underlying rhythm accounts for the difference in mortality. The method with which ventricular arrhythmias lead to the progression of heart failure requires further investigation. It has been recently proposed that it may be a result of delayed myocardial recovery, producing a bioenergetic crisis that leads to a cycle of increasing pump failure and arrhythmias. ${ }^{16}$

\section{ARRHYTHMIA MECHANISMS}

\section{Automaticity}

The normal depolarisation sequence of the heart is set by pacemaker cells, which spontaneously depolarise. Cellular damage and the associated increase in sympathetic activity can disturb the automaticity of pacemaker cells. ${ }^{15}$ Abnormal automaticity has been found in subendocardial Purkinje fibres following ischaemia, which may be a result of underlying abnormalities in calcium handling. ${ }^{19}$
Heart failure disease progression ACC/ AHA stage
B

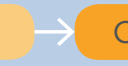

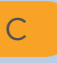

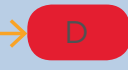

QR

(1)

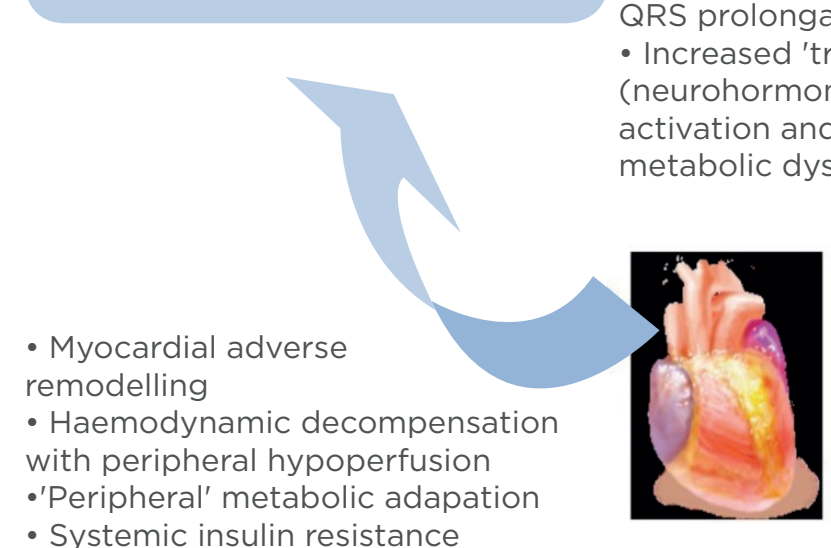

- Myocardial fibrosis

- Progression of arrhythmogenic substrate - Electrical remodeling (Connexin 43, $\mathrm{Ca}^{2+}$ cycling/NCX, and QT and QRS prolongation) - Increased 'triggers' (neurohormonal activation and metabolic dysregulation)
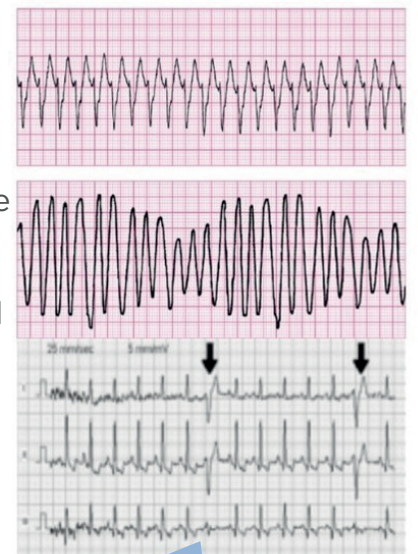

- Dyssynchronous myocardium (RV + LV)

- Mechanical/bioenergetic uncoupling

- Myocardial ('central') metabolic adaptation-shift back to lipids/ketones.

Figure 1: Schema illustrating the pathophysiological cycle of ventricular arrhythmias in advanced heart failure.

ACC/AHA Heart Failure Classification

Stage B: patients with structural heart disease without symptoms of heart failure.

Stage C: patients who have developed clinical heart failure.

Stage D: patients with refractory heart failure requiring specialised interventions.

ACC: American College of Cardiology; AHA: American Heart Association; A-HF: advanced heart failure; LV: left ventricle; RV: right ventricle; VA: ventricular arrhythmia.

Adapted from Santangeli $P$ et al. ${ }^{16}$ 


\section{Triggered Activity}

Triggered activity refers to abnormal potentials triggered by a preceding action potential and includes early and late afterdepolarisations. Myocardial stretch in heart failure has been shown to contribute to increased triggered activity through the reduction in action potential duration. ${ }^{20}$ Early afterdepolarisation manifests when there is a net inward current, which can occur in slow heart rates because of a reduction in the outward $\mathrm{K}^{+}$ currents. ${ }^{21} \quad$ An increase in intracellular $\mathrm{Ca}^{2+}$ concentration accounts for late afterdepolarisations, which ultimately leads to an increased inward $\mathrm{Na}^{+}$current through the activation of the $\mathrm{Ca}^{2+} / \mathrm{Na}^{+}$exchanger. ${ }^{22}$

\section{Re-entry}

Re-entry is the most common and significant mechanism of ventricular arrhythmias in heart failure. It occurs as a result of the disorganisation and slowing of action potential conduction, allowing electrical re-entry circuits to form around regions of scarring. Heart failure contributes to this pathology through neurohormonal pathway disruption, myocardial remodelling and fibrosis, and the disruption of metabolic homeostasis. ${ }^{16}$

In addition to reducing cardiac function and reserve, fibrosis provides an electrophysiological substrate to trigger and sustain arrhythmias. The volume and distribution of scarring has been shown to correlate with ventricular arrhythmias. ${ }^{23}$ Cardiac resynchronisation therapy (CRT) decreases fibrosis and reduces remodelling. ${ }^{24}$ The reversal of cardiac remodelling achieved by CRT has been associated with a reduction in the risk of life-threatening ventricular arrhythmias in cases of mild heart failure. ${ }^{25}$ In contrast, the prevalence of ventricular arrhythmias following implantation of left ventricular assist devices remains high. ${ }^{26}$ Therefore, disease regression in heart failure does not necessarily lead to a reduction in arrhythmias, but it depends on the mechanism through which regression is achieved. Neurohormonal blockade with mineralocorticoid receptor antagonists and angiotensin-converting enzyme inhibitors has also been shown to be effective at reducing the burden of ventricular arrhythmias. ${ }^{27}$

\section{CLINICAL MANIFESTATIONS}

Heart failure is a clinical syndrome characterised by impaired heart function and an inability to pump blood to adequately maintain circulation. Heart failure can be subclassified according to the ventricular ejection fraction: the percentage of ventricular blood ejected with each beat. An ejection fraction $<50 \%$ is considered heart failure with reduced ejection fraction (HFrEF), whereas $>50 \%$ is considered preserved (HFpEF). Approximately $50 \%$ of symptomatic heart failure patients have a preserved ejection fraction. ${ }^{28}$ Patients with HFrEF have a higher prevalence of ventricular arrhythmias and increased risk of sudden death.

The symptoms of heart failure are predominantly due to congestion, which commonly presents as dyspnoea on exertion. ${ }^{10}$ Patients also complain of orthopnoea (breathlessness when lying down) and paroxysmal nocturnal dyspnoea (sudden attacks of breathlessness during the night). Congestion may also occur in the liver, intestines, and peripheries. Signs of heart failure include gallop rhythm with a third heart sound (S3), elevated jugular venous pressure, hepatomegaly, ascites, and peripheral oedema.

Ventricular arrhythmias primarily produce cardiac symptoms, including palpitations, chest pain, dyspnoea, dizziness, syncope, and sudden cardiac death. Ventricular arrhythmia should be suspected in cases of syncope occurring at rest or when lying down.

\section{EVALUATION AND RISK STRATIFICATION}

A detailed history and examination are required when assessing patients with heart failure. Routine laboratory tests and imaging studies can support the diagnosis of heart failure and assist in risk stratification. Although there are a large number of risk factors identified for sudden cardiac death in heart failure, developing a comprehensive risk stratification strategy remains a clinical challenge that requires the analysis of multiple parameters.22,29 Currently, there is no combination of tests that can definitively predict arrhythmic events. However, a depressed ejection fraction and symptoms of heart failure are the most consistent predictors 
of sudden cardiac death. ${ }^{29}$ Indeed, many international guidelines suggest that an ejection fraction $<30 \%$ in NYHA class I, or $<35 \%$ in class II and III, is an indication for ICD implantation in heart failure patients. ${ }^{29}$ Other parameters that may be useful in risk stratification include $T$ wave alternans, signal-averaged ECG, autonomic tone, and electrophysiology studies or ischaemic substrates.

\section{Imaging studies}

Transthoracic echocardiogram is usually the first study undertaken in all patients with suspected heart failure, by virtue of its simplicity and widespread availability. Echocardiogram is central in identifying structural abnormalities, characterising the function of the chambers and valves, and evaluating suitability for further interventions; additionally, the technique can characterise ventricular ejection fraction, wall thickness, wall motion, geometry, and volume. ${ }^{30}$ Serial echocardiograms may be employed to monitor responses to treatment. Echocardiograms are key for differentiating between preserved and reduced ejection fraction heart failure. A reduction in ejection fraction, which is typically considered as an ejection fraction $<50 \%$, has a higher mortality rate and is the most consistent predictor of sudden cardiac death in heart failure. ${ }^{22}$ According to the American College of Cardiology (ACC) and the American Heart Association (AHA), heart failure with preserved ejection fraction can be diagnosed based on clinical symptoms and signs if there is no evidence of valvular abnormality nor impairment of ejection fraction. ${ }^{31}$

Advances in cardiac MRI have permitted novel methods in identifying structural abnormalities and scarring. ${ }^{22}$ Gadolinium-enhanced imaging allows for a detailed analysis of cardiac tissues and identification of areas of tissue scarring. Areas of fibrosis detected on MRI scans have been associated with a significant increase in risk of ventricular arrhythmias and sudden cardiac death. ${ }^{32}$ MRI may also be used to differentiate between heart failure patients with ischaemic and nonischaemic aetiology. ${ }^{33}$

Chest radiography may be used in the work-up for heart failure to exclude alternative conditions and support the diagnosis. Findings of heart failure include cardiomegaly, pulmonary oedema, and pleural effusion.

\section{Electrocardiogram}

The 12 lead ECG in heart failure patients typically shows a variety of abnormalities, although none of them are specific to heart failure.30 Patients may present with QRS complex and $\mathrm{T}$ wave abnormalities, including bundle branch blocks and atrioventricular block. ${ }^{31}$ Underlying ischaemia or myocardial stress can produce changes in the $Q$ waves, $T$ waves, and ST segment of the ECG.30 Remodelling, induced by compensatory mechanisms, can lead to supraventricular or ventricular arrhythmias. ${ }^{34}$ While an ECG cannot predict the risk of sudden cardiac death, it is useful for uncovering conditions which may predispose to arrhythmias, such as WolffParkinson-White syndrome, long QT syndrome, or Brugada syndrome. ${ }^{35}$ Moreover, a recording of VT or premature ventricular complexes can help determine the targets for catheter-based ablation procedures. ${ }^{15}$

The signal-averaged ECG is a high-resolution technique, whereby electrical signals from the heart are averaged with high-gain amplification and filtering, allowing low amplitude deflections in the terminal part of the QRS complex to be detected. ${ }^{36}$ These late potentials reflect the presence of substrate for re-entry and are thought to correlate with areas of delayed endocardial activation. ${ }^{22,37}$ While the negative predictive value of signal-averaged ECG recordings is high for sudden cardiac death, it has a low positive predictive value, which limits its usefulness as a prognostic tool. ${ }^{35,38}$ Ambulatory ECG monitoring can be employed with a Holter monitor, an event monitor, or an implantable loop recorder; however, there is conflicting evidence for their predictive value for ventricular arrhythmias in heart failure. ${ }^{38}$

\section{Biomarkers}

Brain natriuretic peptide (BNP) and N-terminal pro-BNP are blood markers of myocardial stretch that have seen increasing use in evaluating the presence and assessing severity of heart failure. ${ }^{30}$ Cardiac markers of injury, such as troponin I, may be used to ascertain the presence of acute coronary syndrome, although it should be noted that they are commonly elevated in heart failure despite the absence of recent infarction. 


\section{Electrophysiology Studies}

The underlying pathologic substrate and arrhythmic presentation determine the diagnostic and prognostic value of electrophysiological testing. ${ }^{38}$ The inducibility of monomorphic VT with programmed electrical stimulation is a powerful marker for increased risk of ventricular arrhythmias in heart failure patients with reduced ejection fraction and prior myocardial infarction. ${ }^{39}$ In contrast, patients with non-ischaemic aetiology have a much lower inducibility of arrhythmias and the predictive value of these electrophysiological studies remains limited in this population because clinical outcomes do not correlate with inducibility. ${ }^{40}$

\section{MANAGEMENT STRATEGIES}

The initial management of heart failure relies on lifestyle modification and pharmacological therapy. Ventricular arrhythmias should be managed with treatment of the underlying cardiomyopathy, addressing reversible factors, and optimising heart failure status. ${ }^{41}$ ICD provide the best protection against sudden cardiac death from ventricular arrhythmias; however, recurrent ICD shocks are associated with long-term morbidity and mortality. ${ }^{41}$

Consequently, anti-arrhythmic drugs and catheter ablation are important adjuncts for minimising ICD shocks in recurrent ventricular arrhythmias (Figure 2). ${ }^{42}$

\section{Sustained VT or VF in patients with ICD}

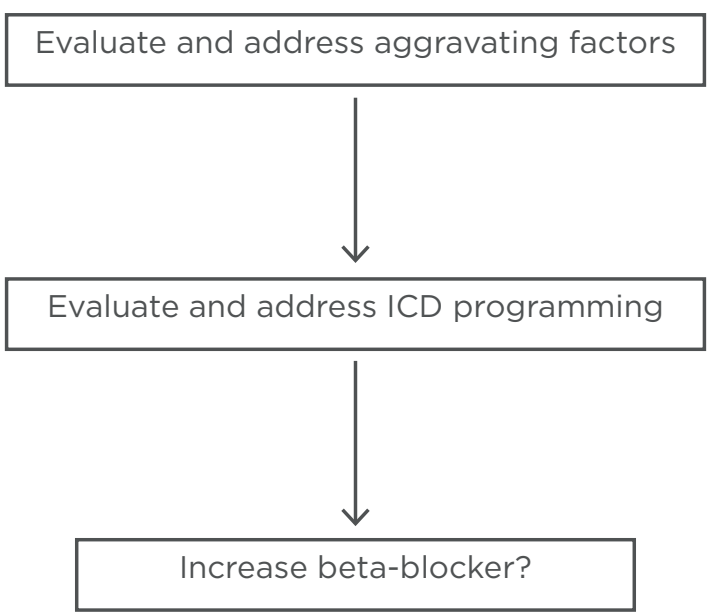

\begin{tabular}{|c|c|}
\hline $\begin{array}{c}\text { Catheter ablation } \\
\text { favoured }\end{array}$ & $\begin{array}{c}\text { Drug therapy } \\
\text { favoured }\end{array}$ \\
\hline
\end{tabular}

- Monomorphic VT

- Experienced centre

- Acceptable procedure risk

- Incessant or very frequent VT

- Increased risk for drug toxicities

- Patient preference

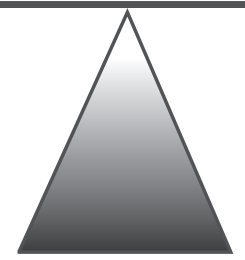

- Polymorphic VT

- Experienced centre not available

- High procedure risk

- Patient preference

Figure 2: Illustration of an approach to the patient with an implantable cardioverter defibrillator who has a ventricular tachycardia recurrence.

ICD: implantable cardioverter defibrillator; VF: ventricular fibrillation; VT: ventricular tachycardia. Adapted from Stevenson WG. ${ }^{42}$ 


\section{Implantable Cardioverter Defibrillator Devices}

ICD therapy effectively terminates ventricular arrhythmias with overdrive (anti-tachycardia) pacing or defibrillation shocks. It is recommended for most heart failure patients with a history of ventricular arrhythmias (secondary prevention) or those that are at an increased risk of sudden death (primary prevention). ${ }^{42}$ Primary prevention is most commonly used in patients exhibiting HFrEF $(\leq 35 \%)$, although it may be recommended in those with genetic diseases that predispose them to sudden death, including hypertrophic cardiomyopathy and Brugada syndrome.

Prophylactic treatment with ICD for VT has been shown to improve prognosis in two large-scale randomised trials that compared ICD therapy with standard medical therapy in patients with predefined risk (MADIT and MUSTT); ${ }^{35}$ however, it is important to note that ICD shocks treat but do not prevent the recurrence of arrhythmias. Recurrent ICD shocks are associated with increased mortality and a reduction in quality of life. ${ }^{41}$ Anti-arrhythmic drugs and catheter ablation should be considered in cases of recurrent arrhythmias. The MADIT-RIT and PREPARE studies evaluated ICD programming strategies to minimise shocks. ${ }^{43,44}$ The strategies that were effective in reducing shocks and improving mortality included anti-tachycardia pacing, increased tachycardia detection rate, delayed-therapy programming, and supraventricular discrimination algorithms.

\section{Pharmacological Agents}

Beta-blockers have been shown to reduce morbidity and mortality in heart failure. ${ }^{41}$ Therefore, given their benefits in improving haemodynamic status, beta-blockers should be offered to all patients without a major contraindication. Furthermore, in cases where patients present with ventricular arrhythmias and ICD shocks who are on optimal beta blocker therapy, the addition of other anti-arrhythmic drugs should be considered.

Anti-arrhythmic drugs are primarily used to minimise and prevent ICD shocks; ${ }^{15}$ nevertheless, there is limited data supporting the efficacy and safety of these drugs in patients with heart failure. ${ }^{45}$ While they may decrease arrhythmic burden, many anti-arrhythmic drugs have negative inotropic effects that can lead to the worsening of haemodynamic status. In particular, there is very little data on the efficacy and safety of shortterm pharmacological management of recurrent VT. Intravenous lignocaine is used because of its short half-life and good safety profile, ${ }^{46}$ but intravenous amiodarone may be considered. Long-term management with negatively inotropic agents, such as sotalol and procainamide, should be avoided.

Most class I anti-arrhythmic agents, which act by blocking the $\mathrm{Na}^{+}$channel, have been shown to increase mortality in structural heart disease patients because of the negative inotropic effect and potential proarrhythmic action. ${ }^{47}$ Nevertheless, quinidine and mexiletine have some evidence of effective anti-arrhythmic action, although they have never been evaluated in randomised controlled trials. ${ }^{48,49}$ It should be noted that mexiletine should be used with caution because it may worsen haemodynamic status in advanced heart failure through its negative inotropic and increased peripheral vascular resistance effects..$^{50}$

Randomised control trials have shown that amiodarone is the only class III anti-arrhythmic drug with a significant treatment effect on ventricular arrhythmias in advanced heart failure. ${ }^{45} \mathrm{~A}$ systematic review and meta-analysis ${ }^{45}$ on the effectiveness of anti-arrhythmic drugs for the prevention of recurrent ventricular arrhythmias analysed eight trials, which showed that amiodarone significantly reduced appropriate ICD interventions (odds ratio $=0.3$; $\mathrm{p}<0.001$ ), while sotalol did not have a significant impact (odds ratio=0.83; $p=0.594$ ); however, amiodarone is associated with end-organ toxicity, significant side-effects, and a possible increase in all-cause mortality. ${ }^{51,52}$

Class IV drugs non-dihydropyridine $\mathrm{Ca}^{2+}$ channel blockers should be avoided as they have poor efficacy in ventricular arrhythmias on a background of heart failure, and may increase overall mortality. ${ }^{41}$ Analysis of 15 randomised trials has shown that using amiodarone has an unfavourable profile with a number needed to treat of 38 for prophylaxis of sudden cardiac death, compared to the number needed to harm of 14 from amiodarone-associated thyroid toxicity, hepatic toxicity, pulmonary toxicity, or bradycardia. ${ }^{51}$ 

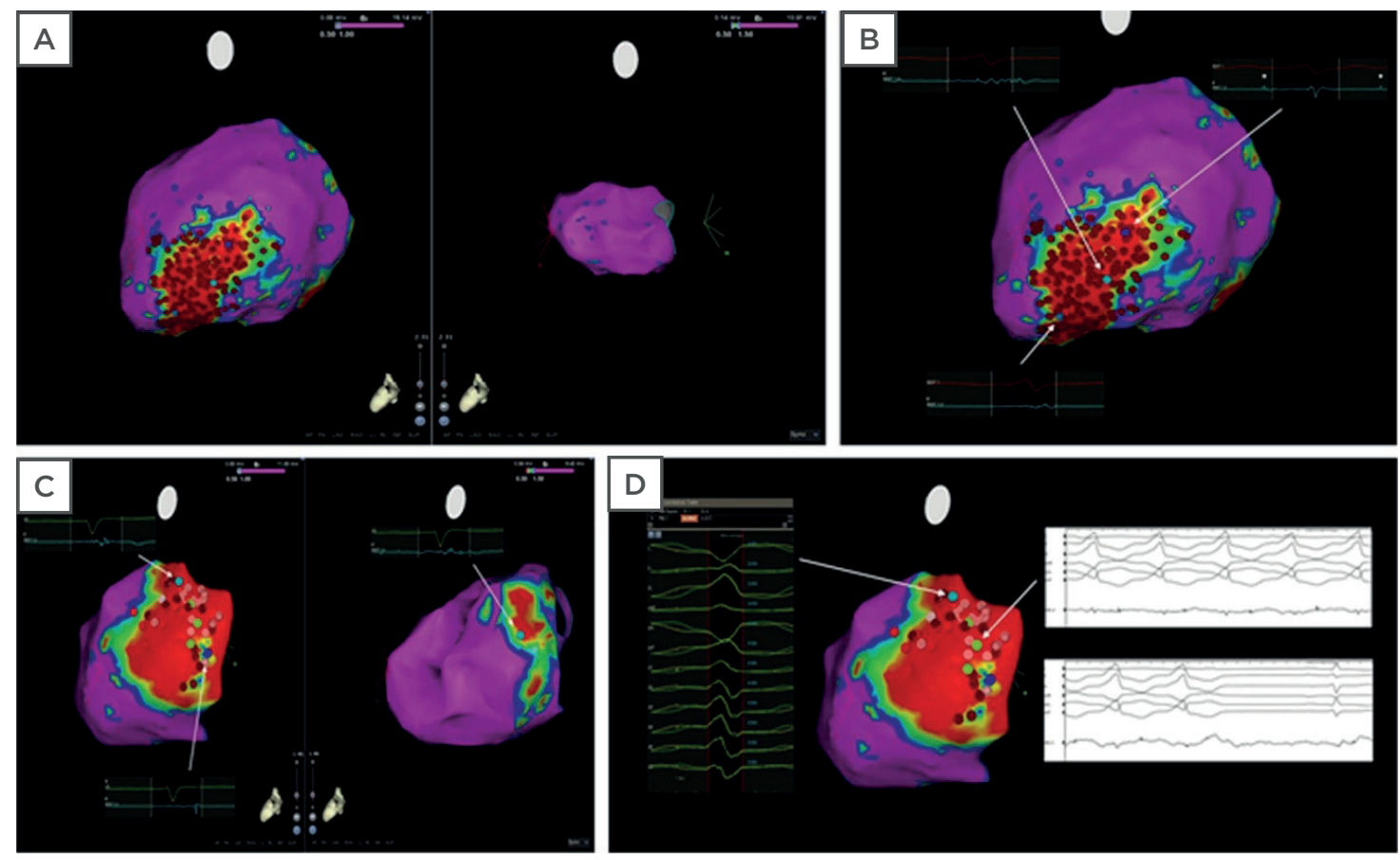

Figure 3: Electroanatomic maps of sample ablation lesions.

Electroanatomic maps ( $A, B$ ) showing an example of extensive radiofrequency catheter ablation (red dots) in the scar zone (i.e., 'scar homogenisation') in a patient with ventricular tachycardia, nonischaemic cardiomyopathy, and epicardial scar. Normal voltage was present in the endocardium (A; right panel), while scar was present in the epicardium. In a different patient, maps show limited ablation performed in the epicardial scar zone (C), with mapping and termination of the ventricular arrhythmia during ablation (D).

Colours: Red regions represent scar tissue (bipolar voltage $<0.5 \mathrm{mV}$ ); purple regions represent normal myocardium (bipolar voltage >1.5 mV); other coloured regions represent 'border zones' (bipolar voltage between 0.5 and $1.5 \mathrm{mV}$ ).

Dots: Light blue = fragmented potentials; dark blue = late potential; light and dark red = ablation sites; and green = sites of VT termination during radiofrequency ablation.

Adapted from Gökoğlan $Y$ et al. ${ }^{53}$

\section{Catheter-Based Interventions}

Catheter ablation can be used as an adjunctive treatment in patients with frequent ventricular arrhythmias and ICD shocks despite pharmacological therapy. The benefits of this intervention depends on the location of the circuit, the underlying substrate, arrhythmia inducibility and NYHA heart failure status (Figure 3). ${ }^{15,53}$ A recent large single-centre study reported that arrhythmia-free survival rates, at a median of 6 years post-VT ablation, were 54\% $\pm 4 \%$ in patients with ischaemic cardiomyopathy and $38 \% \pm 4 \%$ in non-ischaemic cardiomyopathy. ${ }^{54}$ Earlier adoption of ablation strategies in post infarct VT has been associated with better outcomes. ${ }^{55}$

Catheter ablation is the most effective monomorphic VT therapy, although it has been shown to be efficacious in some cases of polymorphic $\mathrm{VT}$ and $\mathrm{VF}^{42}$ The central isthmus, an essential component of the re-entry circuit, is usually targeted in monomorphic VT;56 however, haemodynamic instability often hinders the ability to effectively map the circuit. In such cases, scars can be defined during sinus rhythm using voltage mapping as the central isthmus is usually found between areas of dense scarring. ${ }^{57}$ While the acute success rate in abolishing VT using ablation is approximately $70 \%$, at 12 month of follow-up, $26-50 \%$ of patients will experience recurrence of $\mathrm{VT} .{ }^{42}$ There is limited data on the use of catheter ablation for $\mathrm{VT}$ in severe heart failure because of the increased mortality risk and safety concerns.

A recent paper reported that radiofrequency ablation of VT in NYHA class IV patients can 
be safely performed despite the higher rate of comorbidities and reduces mortality. ${ }^{58}$ It reported that NYHA class IV patients without recurrent $\mathrm{VT}$ had similar survival rates to NYHA class II and III patients with recurrent VT.

\section{Autonomic modulation}

Sympathetic stimulation and parasympathetic downregulation contribute to the compensatory mechanisms that lead to cardiac remodelling and fibrosis. Therefore, 'neuraxial modulation', targeting the autonomic nervous system has provided a novel therapeutic strategy for managing refractory ventricular arrhythmias..$^{16,59}$ In a study of 14 patients with a mean followup of 6 months, left cardiac sympathetic denervation (stellate ganglionectomy) or thoracic epidural anaesthesia resulted in a significant reduction in ICD shocks, and 48\% of patients had complete suppression of ventricular arrhythmias. ${ }^{59}$ It has been shown that renal denervation can significantly reduce VT burden. ${ }^{60}$ A recent multicentre study reported a significant reduction in the burden of ICD shocks in patients undergoing left or bilateral cardiac sympathetic denervation.61 At a median follow-up of 1.1 years, ICD shocks reduced from a mean of $18 \pm 30$ to $2.0 \pm 4.3$. Factors associated with less successful outcomes included advanced heart failure, longer VT cycle lengths, and left-sided only denervation.

\section{CONCLUSION}

The increasing age of the worldwide population is contributing to a growing incidence of heart failure. The consequent increase in ventricular arrhythmias represents a significant cause of morbidity and mortality. The pathophysiology underlying the interaction between heart failure and arrhythmias is complex; each condition may predispose to or worsen the other. Technological advances in imaging and biomarkers aid early diagnosis, risk stratification, and treatment. ICD device implantation has been the focus of clinical management of ventricular arrhythmias in recent decades given the strong evidence of efficacy; however, there is also evidence for the role of pharmacological therapy and invasive catheter ablation procedures. The outcomes of different management strategies for ventricular arrhythmias in heart failure requires further elucidation with further prospective studies.

\section{References}

1. Mosterd A et al. The prognosis of heart failure in the genera population: The Rotterdam Study. Eur Heart J. 2001;22(15):1318-27.

2. Saltzman HE. Arrhythmias and heart failure. Cardiol Clin. 2014;32(1):125-33.

3. Myerburg RJ et al. Survivors of prehospital cardiac arrest. JAMA. 1982;247(10):1485-90

4. Bristow MR et al.; Comparison of Medical Therapy, Pacing, and Defibrillation in Heart Failure (COMPANION) Investigators. Cardiacresynchronization therapy with or without an implantable defibrillator in advanced chronic heart failure. N Engl J Med. 2004;350(21):2140-50.

5. Bardy GH et al.; Sudden Cardiac Death in Heart Failure Trial (SCDHeFT) Investigators. Amiodarone or an implantable cardioverterdefibrillator for congestive heart failure. $\mathrm{N}$ Engl J Med 2005;352(3):225-37

6. Ambrosy AP et al. The global health and economic burden of hospitalizations for heart failure: Lessons learned from hospitalized heart failure registries. J Am Coll Cardiol. 2014;63(12):1123-33.

7. Mosterd A Hoes AW. Clinical epidemiology of heart failure. Heart. 2007:93(9):1137-46.

8. Kjekshus J. Arrhythmias and mortality in congestive heart failure. Am J Cardiol. 1990;65(19):421-81.

9. Sweeney MO. Sudden death in heart failure associated with reduced left ventricular function: Substrates, mechanisms, and evidence-based management, Part II. Pacing and clinical electrophysiology. Pacing Clin Electrophysiol. 2001;24(6):1002-22.

10. Luu M et al. Diverse mechanisms of unexpected cardiac arrest in advanced heart failure. Circulation 1989;80(6):1675-80.

11. Gupta S, Figueredo VM. Tachycardia mediated cardiomyopathy:

Pathophysiology, mechanisms, clinical features and management. Int $\mathrm{J}$ Cardiol. 2014;172(1):40-6.

12. Lip GYH et al. European Heart Rhythm Association/Heart Failure Association joint consensus document on arrhythmias in heart failure, endorsed by the Heart Rhythm Society and the Asia Pacific Heart Rhythm Society. EP Europace. 2016;18(1):12-36.

13. Kemp CD, Conte JV. The pathophysiology of heart failure. Cardiovasc Pathol. 2012;21(5):365 71

14. Tomaselli GF, Rose J. Molecular aspects of arrhythmias associated with cardiomyopathies. Curr Opin Cardiol. 2000;15(3):202-8.

15. Gillespie HS et al. Arrhythmias in structural heart disease. Curr Cardiol Rep. 2014;16(8):510.

16. Santangeli $P$ et al. Management of ventricular arrhythmias in patients with advanced heart failure. J Am Coll Cardiol. 2017;69(14):1842-60.

17. Saxon LA et al. Predictors of sudden cardiac death and appropriate shock in the Comparison of Medical Therapy, Pacing, and Defibrillation in Heart Failure (COMPANION) Trial. Circulation. 2006;114(25):2766-72.

18. Powell BD et al. Survival after shock therapy in implantable 
cardioverter-defibrillator and cardiac resynchronization therapydefibrillator recipients according to rhythm shocked. The ALTITUDE survival by rhythm study. J Am Coll Cardiol. 2013;62(18):1674-9.

19. Pogwizd SM, Bers DM. Calcium cycling in heart failure: The arrhythmia connection. J Cardiovasc Electrophysiol. 2002;13(1):88-91.

20. Dean JW, Lab MJ. Arrhythmia in hear failure: Role of mechanically induced changes in electrophysiology. Lancet. 1989;1(8650):1309-12

21. Zeng J, Rudy Y. Early afterdepolarizations in cardiac myocytes: Mechanism and rate dependence. Biophys J. 1995;68(3):949-64.

22. Lo R, Hsia HH. Ventricular arrhythmias in heart failure patients. Cardiol Clin. 2008;26(3):381-403.

23. Yokokawa $M$ et al. The characteristics and distribution of the scar tissue predict ventricular tachycardia in patients with advanced heart failure. Pacing Clin Electrophysiol. 2009;32(3):314-22.

24. D'Ascia C et al. Effects of biventricular pacing on interstitial remodelling, tumor necrosis factoralpha expression, and apoptotic death in failing human myocardium. Eur Heart J. 2006;27(2):201-6.

25. Barsheshet A et al. Reverse remodeling and the risk of ventricular tachyarrhythmias in the MADIT-CRT (Multicenter Automatic Defibrillator Implantation Trial-Cardiac Resynchronization Therapy). J Am Coll Cardiol. 2011;57(24):2416-23.

26. Garan AR et al. Early post-operative ventricular arrhythmias in patients with continuous-flow left ventricular assist devices. J Heart Lung Transplant. 2015;34(12):1611-6.

27. Pitt B et al. Eplerenone reduces mortality 30 days after randomization following acute myocardial infarction in patients with left ventricular systolic dysfunction and heart failure. J Am Coll Cardiol. 2005;46(3):425-31.

28. Go AS et al.; American Heart Association Statistics Committee and Stroke Statistics Subcommittee. Heart disease and stroke statistics--2013 update: A report from the American Heart Association. Circulation. 2013;127(1):e6-e245

29. Masarone D et al. Management of arrhythmias in heart failure. $J$ Cardiovasc Dev Dis. 2017;4(1):3.

30. Patel RB, Secemsky EA. Clinical features of heart failure and acute coronary syndromes. Clin Lab Med. 2014;34(1):15-30.

31. Yancy CW et al.; American College of Cardiology Foundation/ American Heart Association Task Force on Practice Guidelines. 2013 ACCF/AHA guideline for the management of heart failure: A report of the American College of Cardiology Foundation/American Heart Association Task Force on Practice Guidelines. Circulation. 2013;128(16):e240-327.

32. Assomull RG et al. Cardiovascular magnetic resonance, fibrosis, and prognosis in dilated cardiomyopathy. J Am Coll Cardiol. 2006;48(10):197785.

33. Assomull RG et al. Cardiovascular magnetic resonance in the evaluation of heart failure. Heart. 2007;93(8):985-92.

34. Wang CS et al. Does this dyspneic patient in the emergency department have congestive heart failure? JAMA 2005;294(15):1944-56.

35. Huikuri HV et al. Sudden death due to cardiac arrhythmias. N Engl J Med. 2001;345(20):1473-82.

36. Borggrefe $M$ et al. Prediction of arrhythmia risk based on signalaveraged ECG in postinfarction patients. Pacing Clin Electrophysiol. 1997:20(10 Pt 2):2566-76.

37. Vassallo JA et al. Relation of late potentials to site of origin of ventricular tachycardia associated with coronary heart disease. Am J Cardiol. 1985;55(8):985-9.

38. Klein L, Hsia H. Sudden cardiac death in heart failure. Cardiol Clin. 2014;32(1):135-44.

39. Naccarella F et al. Arrhythmic risk stratification of post-myocardial infarction patients. Curr Opin Cardiol. 2000;15(1):1-6.

40. Hsia HH, Marchlinski FE. Electrophysiology studies in patients with dilated cardiomyopathies. Card Electrophysiol Rev. 2002;6(4):472-81.

41. Sengupta J, Abdelhadi R. Approach to reduction of ventricular arrhythmias and implantable cardioverter-defibrillator therapies in patients with heart failure. Curr Opin Cardiol. 2013;28(3):337-43

42. Stevenson WG. Current treatment of ventricular arrhythmias: State of the art. Heart Rhythm. 2013;10(12):191926.

43. Moss AJ et al.; MADIT-RIT Tria Investigators. Reduction in inappropriate therapy and mortality through ICD programming. N Engl Med. 2012:367(24):2275-83.

44. Wilkoff BL et al.; PREPARE Study Investigators. Strategic programming of detection and therapy parameters in implantable cardioverter-defibrillators reduces shocks in primary prevention patients: Results from the PREPARE (Primary Prevention Parameters Evaluation) study. J Am Coll Cardiol. 2008;52(7):541-50.

45. Santangeli $P$ et al. Comparative effectiveness of antiarrhythmic drugs and catheter ablation for the prevention of recurrent ventricular tachycardia in patients with implantable cardioverterdefibrillators: A systematic review and meta-analysis of randomized controlled trials. Heart Rhythm. 2016;13(7):1552-9.

46. Rademaker AW et al. Character of adverse effects of prophylactic lidocaine in the coronary care unit. Clin Pharmacol Ther. 1986;40(1):71-80.

47. Stevenson WG et al. Improving survival for patients with atrial fibrillation and advanced heart failure. J Am Coll Cardiol. 1996;28(6):145863. Erratum in: J Am Coll Cardiol. 1997;30(7):1902.

48. Hoffmeister HM et al. Negative inotropic effect of class-Iantiarrhythmic drugs: Comparison of flecainide with disopyramide and quinidine. Eur Heart J. 1987;8(10):112632.

49. Luderitz $B$ et al. Combination of antiarrhythmic drugs. J Cardiovasc Pharmacol. 1991;17 Suppl 6:S48-52.

50. Gottlieb SS, Weinberg M. Cardiodepressant effects of mexiletine in patients with severe left ventricular dysfunction. Eur Heart J. 1992;13(1):22-7.

51. Santangeli $P$ et al. Examining the safety of amiodarone. Expert Opin Drug Saf. 2012;11(2):191-214.

52. Connolly SJ et al.; Optimal Pharmacological Therapy in Cardioverter Defibrillator Patients (OPTIC) Investigators. Comparison of beta-blockers, amiodarone plus betablockers, or sotalol for prevention of shocks from implantable cardioverter defibrillators: The OPTIC Study: A randomized trial. JAMA. 2006:295(2):165-71.

53. Gökoğlan $Y$ et al. Scar homogenization versus limitedsubstrate ablation in patients with nonischemic cardiomyopathy and ventricular tachycardia. J Am Coll Cardiol. 2016;68(18):1990-8.

54. Kumar S et al. Long-term outcomes after catheter ablation of ventricular tachycardia in patients with and without structural heart disease. Heart Rhythm. 2016;13(10):1957-63.

55. Reddy $\vee Y$ et al. Prophylactic catheter ablation for the prevention of defibrillator therapy. N Engl J Med. 2007:357(26):2657-65.

56. Zeppenfeld K, Stevenson WG. Ablation of ventricular tachycardia in patients with structural heart disease. Pacing Clin Electrophysiol. 2008:31(3):358-74

57. Sasaki T et al. Myocardial structural associations with local electrograms: A study of postinfarct ventricular tachycardia pathophysiology and magnetic resonance-based noninvasive mapping. Circ Arrhythm Electrophysiol. 2012;5(6):1081-90. 
58. Tzou WS et al. Ventricular tachycardia ablation in severe heart failure: An international ventricular tachycardia ablation center collaboration analysis.

59. Bourke T et al. Neuraxial modulation for refractory ventricular arrhythmias:
Value of thoracic epidura anesthesia and surgical left cardiac sympathetic denervation. Circulation. 2010;121(21):2255-62.

60. Bradfield JS et al. Renal denervation for refractory ventricular arrhythmias. Trends Cardiovasc Med. 2014;24(5):206-13.

61. Vaseghi $\mathrm{M}$ et al. Cardiac sympathetic denervation for refractory ventricular arrhythmias. J Am Coll Cardiol. 2017;69(25):3070-80. 